\title{
Hypercholesterolemia decreases nitric oxide production by promoting the interaction of caveolin and endothelial nitric oxide synthase
}

\author{
Olivier Feron, Chantal Dessy, Stephane Moniotte, Jean-Pierre Desager, \\ and Jean-Luc Balligand
}

Department of Medicine, Unit of Pharmacology and Therapeutics, Université catholique de Louvain, B-1200 Brussels, Belgium

Address correspondence to: Jean-Luc Balligand, Department of Medicine, Unit of Pharmacology and Therapeutics, FATH 5349, Université catholique de Louvain, Avenue E. Mounier, 53, B-1200 Brussels, Belgium. Phone: 32-2-764-9326; Fax: 32-2-764-9322; E-mail: balligand@mint.ucl.ac.be

Received for publication August 5, 1998, and accepted in revised form January 26, 1999.

\begin{abstract}
Hypercholesterolemia is a central pathogenic factor of endothelial dysfunction caused in part by an impairment of endothelial nitric oxide (NO) production through mechanisms that remain poorly characterized. The activity of the endothelial isoform of NO synthase (eNOS) was recently shown to be modulated by its reciprocal interactions with the stimulatory $\mathrm{Ca}^{2+}$-calmodulin complex and the inhibitory protein caveolin. We examined whether hypercholesterolemia may reduce NO production through alteration of this regulatory equilibrium. Bovine aortic endothelial cells were cultured in the presence of serum obtained from normocholesterolemic (NC) or hypercholesterolemic (HC) human volunteers. Exposure of endothelial cells to the HC serum upregulated caveolin abundance without any measurable effect on eNOS protein levels. This effect of HC serum was associated with an impairment of basal NO release paralleled by an increase in inhibitory caveolin-eNOS complex formation. Similar treatment with HC serum significantly attenuated the NO production stimulated by the calcium ionophore A23187. Accordingly, higher calmodulin levels were required to disrupt the enhanced caveolin-eNOS heterocomplex from HC serum-treated cells. Finally, cell exposure to the low-density lipoprotein (LDL) fraction alone dose-dependently reproduced the inhibition of basal and stimulated NO release, as well as the upregulation of caveolin expression and its heterocomplex formation with eNOS, which were unaffected by cotreatment with antioxidants. Together, our data establish a new mechanism for the cholesterol-induced impairment of NO production through the modulation of caveolin abundance in endothelial cells, a mechanism that may participate in the pathogenesis of endothelial dysfunction and the proatherogenic effects of hypercholesterolemia.
\end{abstract}

J. Clin. Invest. 103:897-905 (1999).

\section{Introduction}

Hypercholesterolemia is a causal factor of atherosclerosis, a process histologically characterized by lesions progressing from fatty streaks to fibrous plaques, ultimately occluding the lumen of the affected artery $(1,2)$. Before the appearance of any ultrastructural change in the vessel wall, however, chronic elevations in serum cholesterol are often associated with an impaired endothelium-dependent vasodilation $(3,4)$. This primitive hallmark of endothelial dysfunction is thought to result from an imbalance between the influence of vasodilator and vasoconstrictor autacoids involved in the regulation of vascular tone. Thus, defects in the L-arginine/nitric oxide (NO)/cyclic guanosine monophosphate (cGMP) pathway have progressively emerged as an initiating step in the proatherogenic alteration of the vascular endothelium, leading to the unopposed influence of thrombogenic and proliferative factors on the vessel wall (5-10). While initial studies suggested that native low-density lipoprotein (LDL) might inhibit endothelium-dependent relaxation through inactivation of NO $(11,12)$, oxidized forms of LDL were recently demonstrated to specifically impair NO-dependent arterial relaxation through a variety of mechanisms, including a decrease in endothelial nitric oxide synthase (eNOS) expression, the uncoupling of $\mathrm{G}_{\mathrm{i}}$ protein-dependent signal transduction, or a reduction in eNOS substrate availability (reviewed in refs. 9, 10). However, because most of these studies were done with high concentrations of LDL chemically oxidized in vitro, the relevance of any of these different mechanisms to the pathogenesis of endothelial dysfunction remains controversial $(13,14)$.

In peripheral cells, exposure to elevated plasma cholesterol results in the selective uptake of free cholesterol (FC) from native LDL and in the stimulation of FC efflux (15). Discrete plasmalemmal microdomains termed caveolae were recently shown to actively increase the rate and specificity of FC efflux to high-density lipoprotein (HDL) (reviewed in ref. 15). Moreover, Fielding and coworkers $(16,17)$ have recently reported that in human fibroblasts, the cellular FC level regulates the transcription of the gene encoding caveolin, the structural protein of caveolae. Interestingly, the recent discovery that eNOS is compartmentalized into caveolae $(18,19)$ introduced a new framework for the understanding of the molecular mechanisms whereby exposure to high LDL cholesterol (LDL-C) may alter NO production. Specific interactions between the 
cholesterol-binding protein caveolin and signal-transducing proteins, including eNOS and various kinases, are known to repress the catalytic activity of these enzymes (reviewed in ref. 20). Accordingly, the activity of eNOS was shown to be regulated through its reversible inhibitory interaction with caveolin $(19,21-23)$, a process that could be competed by calmodulin (CaM) in the presence of $\mathrm{Ca}^{2+}$ $(21,24,25)$. As a corollary, the activity of eNOS in endothelial cells can theoretically be influenced by changes in the equilibrium between these two protein-protein interactions upon stoichiometric changes in the abundance of either of these partners. We therefore postulated that exposure to high cholesterol concentrations could decrease NO production through an upregulation of caveolin abundance that would enhance its inhibitory heterocomplex formation with eNOS and attenuate its activation by $\mathrm{Ca}^{2+}$-mobilizing agonists.

To test these hypotheses, we incubated endothelial cells with human serum from normocholesterolemic (NC) and hypercholesterolemic (HC) volunteers, as well as with the different purified lipoprotein subfractions. Through combined measurements of caveolin-eNOS interaction (by coimmunoprecipitation) and $\mathrm{NO}$ production in intact cells (using an NO-selective microprobe) in resting conditions and after agonist stimulation, we established a quantitative correlation between serum cholesterol level, caveolin-eNOS association, and impaired NO release. Finally, we identified serum LDL-C as the subfraction triggering the upregulation of caveolin abundance that leads to the stabilization of its complex formation with eNOS and to inactivation of the enzyme.

\section{Methods}

Human serum and cell culture. Blood was collected from hyperlipemic patients and normolipemic donors who had fasted overnight, and plasma was obtained by centrifugation $(2,000 \mathrm{~g}$, $10 \mathrm{~min}, 2-4^{\circ} \mathrm{C}$ ). None of the hyperlipemic (or normolipemic) subjects had received any hypolipemiant drug in the past 6 weeks before blood collection. Lipoprotein subfractions and lipoprotein-deprived serum (LPDS) were isolated by density gradient ultracentrifugation and dialyzed overnight as described (26). Cholesterol levels of total plasma and lipoprotein subfractions were routinely determined using colorimetric methods according to the manufacturer's instructions (CHOL and HDL-C; Boehringer Mannheim, Mannheim, Germany). Freshly prepared serum and lipoprotein subfractions from different donors were always used in separate experiments.

Bovine aortic endothelial cells (BAEC) were cultured to $60 \%$ confluence in $3.5-\mathrm{cm}$ dishes in DMEM containing $10 \%$ FBS and were serum-starved for $24 \mathrm{~h}$. Cell monolayers were then transferred to $50 \%$ (vol/vol) human serum in DMEM for $48 \mathrm{~h}$; the resulting dilution approximates the lipoprotein concentration to which endothelial cells are exposed in vivo. In some experiments, native serum was replaced by LPDS resupplemented with one of the lipoprotein subfractions. In others, incubations with $\mathrm{NC}$ or $\mathrm{HC}$ serum or LDL-containing medium were carried out in the presence of $25 \mu \mathrm{M} \mathrm{N}$-acetyl-leu-leu-norleucinal (ALLN) (Boehringer Mannheim), a cysteine protease inhibitor, or antioxidants such as $50 \mu \mathrm{M}$ diethylenetriamine pentaacetic acid (DTPA), $20 \mu \mathrm{M}$ butylated hydroxytoluene (BHT), or $100 \mu \mathrm{g} / \mathrm{ml} \mathrm{Cu} / \mathrm{Zn}$ superoxide dismutase (SOD); in the latter experiments, a stock lipoprotein-containing medium was used to replace the incubation medium every 6-8 h. In addition, aliquots of freshly prepared or incubated lipoproteincontaining medium were assayed by ELISA (27) for the presence of malondialdehyde-modified LDL, which remained below detection levels in all cases $(<0.1 \mathrm{mg} / \mathrm{dl})$.

Coimmunoprecipitation and immunoblotting. Endothelial cells were extensively washed with PBS, harvested, pelleted by centrifugation, resuspended in the octylglucoside (OG) buffer containing $60 \mathrm{mM} \mathrm{OG}, 50 \mathrm{mM}$ Tris- $\mathrm{HCl}$ ( $\mathrm{pH}$ 7.4), $125 \mathrm{mM} \mathrm{NaCl}, 2$ $\mathrm{mM}$ dithiothreitol, $100 \mu \mathrm{M}$ EGTA, and protease inhibitors (1 $\mathrm{mg} / \mathrm{ml}$ leupeptin, $1 \mathrm{mg} / \mathrm{ml}$ pepstatin, and $1 \mathrm{mM}$ phenymethysulfonyl fluoride), and sonicated as described previously (19). Aliquots of cell homogenates were incubated with a rabbit caveolin-1 polyclonal antibody (lot 5; Transduction Labs, Lexington, Kentucky, USA). After $1 \mathrm{~h}$ at $4^{\circ} \mathrm{C}$, protein G-Sepharose beads (50 $\mu \mathrm{l}$ of a $50 \%$ slurry) were added to the supernatant for a further 1 $\mathrm{h}$ incubation at $4^{\circ} \mathrm{C}$. Bound immune complexes were washed three times with OG buffer and then once with $50 \mathrm{mM}$ Tris-HCl (pH 7.4), $150 \mathrm{mM} \mathrm{NaCl}$. In some experiments, the supernatant fraction (remaining after pelleting of the protein G-Sepharose immune complexes) was immunoprecipitated by a rabbit anti- a

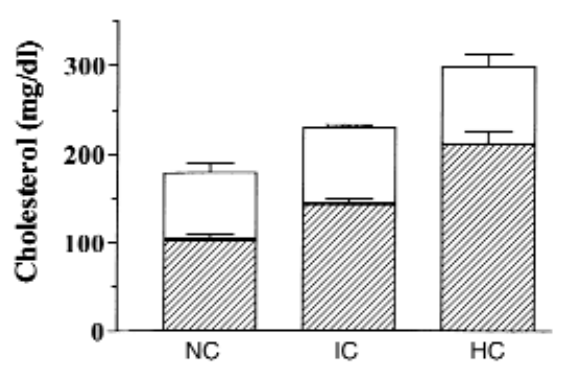

$b$

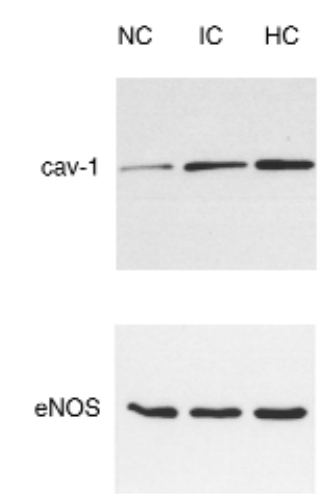

$c$

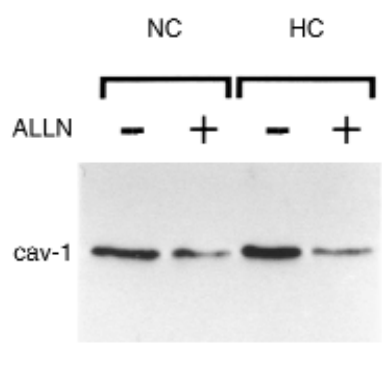

\section{Figure 1}

Caveolin and eNOS expression in endothelial cells exposed to normocholesterolemic and hypercholesterolemic human serum. $(\boldsymbol{a})$ Mean $( \pm$ SEM, $n=10)$ cholesterol concentrations of human plasma fractions divided into three categories and used in the experiments discussed below. Hatched areas correspond to LDL-C as a fraction of total plasma cholesterol. (b) Caveolin (top) and eNOS (bottom) immunoblots from endothelial cells exposed to the corresponding fractions of human plasma. This experiment was repeated four times with equivalent results. (c) The caveolin immunoblots from endothelial cells exposed to $\mathrm{NC}$ or $\mathrm{HC}$ serum in the presence or absence of the cysteine protease inhibitor ALLN ( $25 \mu \mathrm{M})$. This experiment was repeated two times with equivalent results. $A L L N, N$-acetyl-leu-leu-norleucinal; $e N O S$, endothelial nitric oxide synthase; $H C$, hypercholesterolemic; $I C$, intermediate; $L D L-C$, low-density lipoprotein cholesterol; NC, normocholesterolemic. 
a

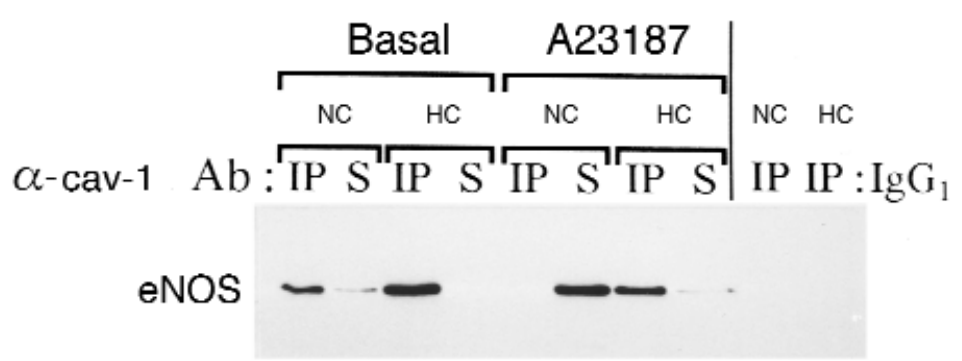

$\boldsymbol{b}$

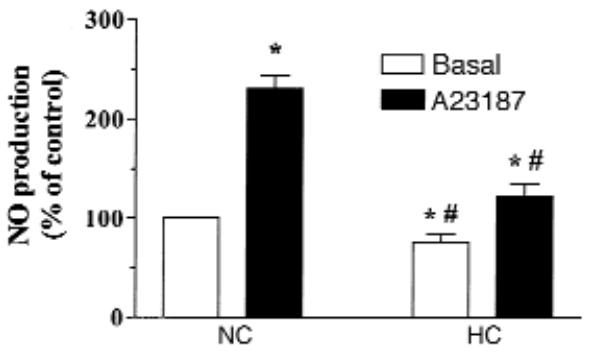

\section{Figure 2}

High plasma cholesterol levels stabilize the caveolin-eNOS interaction and decrease NO production in endothelial cells. Endothelial cells exposed to normocholesterolemic (NC; $179 \pm 10 \mathrm{mg} / \mathrm{dl}$ ) or hypercholesterolemic (HC; $298 \pm 14 \mathrm{mg} / \mathrm{dl}$ ) human serum were incubated for $5 \mathrm{~min}$ in the presence or absence of the calcium ionophore A23187 (5 $\mu \mathrm{M})$ and either tested for NO production or collected, lysed, and solubilized as described in the text.

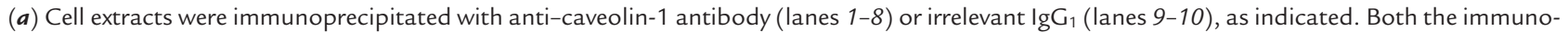
precipitate and supernatant fractions were then separated on SDS-PAGE and immunoblotted with two different antibodies. Top: Immunoblot with an anti-eNOS antibody of caveolin immunoprecipitates $(I P)$ and of the remaining supernatant $(S)$. Note that to obtain the $S$ fraction, the supernatants from the IP were immunoprecipitated with eNOS antibody in order to detect quantitatively the amount of eNOS left behind the initial caveolin IP. Note also that the sum of eNOS protein detected in the caveolin IP and supernatant is equal for the different conditions. Bottom: Immunoblot with an anti-caveolin-1 antibody of caveolin immunoprecipitates (IP) and of the remaining supernatant fractions (S). Note that to obtain the S fractions, the supernatants from the caveolin IP were again immunoprecipitated with anti-caveolin-1 antibody in order to detect quantitatively the amount of caveolin left behind the initial IP. These experiments were performed three times with similar results. $(\boldsymbol{b})$ A bar graph (mean \pm SEM, $n=9)$ illustrating the NO production (measured with a microsensor) at the basal level (open bars) and after exposure to A23187 (filled bars) in cultures of endothelial cells treated with NC or HC human serum; the data are expressed as percent of NO production in nonstimulated cells exposed to NC serum. ${ }^{*} P<0.05$ vs. basal, NC condition. ${ }^{*} P<0.01$ vs. stimulated NC condition. NO, nitric oxide.

eNOS or anti-caveolin-1 polyclonal antibody (Transduction Labs). The immunoprecipitates were eluted by boiling in Laemmli sample buffer. Protocols for SDS-PAGE on 7.5\%-12.5\% polyacrylamide gels, immunoblotting with eNOS or caveolin antibodies (Transduction Labs), and chemiluminescent detection were performed as described previously (19).

NO detection and eNOS activity. BAEC grown on gelatin-coated $35-\mathrm{mm}$ dishes were preincubated in Krebs-Ringer buffer (adjusted to $\mathrm{pH} 7.4$ ) composed of $136 \mathrm{mM} \mathrm{NaCl}, 5.4 \mathrm{mM} \mathrm{KCl}$, $1.8 \mathrm{mM} \mathrm{CaCl}_{2}, 0.8 \mathrm{mM} \mathrm{MgSO}_{4}, 1.0 \mathrm{mM} \mathrm{Na}_{2} \mathrm{HPO}_{4}, 10 \mathrm{mM}$ HEPES, and $55 \mathrm{mM}$ glucose. The buffer was supplemented with $7.5 \mathrm{U} / \mathrm{ml}$ SOD (Sigma Chemical Co., St. Louis, Missouri, USA). NO release was monitored with an NO-selective microprobe (World Precision Instruments; Aston, United Kingdom), as used previously by our group (28). A micromanipulator attached to the stage of an inverted microscope allowed positioning of the probe sensor (200- $\mu \mathrm{m}$ diameter) $\sim 0.1 \mathrm{~mm}$ above the cell surface. NO production was measured at 5 -min intervals in the presence or absence of $5 \mu \mathrm{M} \mathrm{A} 23187$ in a fixed volume of buffer, normalized for the amount of protein in the dish, and expressed as the amount of NO produced per minute and milligram of protein; adequate controls using either vehicle or NOS inhibitors are routinely performed in parallel, as described previously $(28,29)$. Calibration of the electrode was performed using different concentrations of the NO donor Snitroso- $N$-acetyl-DL-penicillamine (SNAP), as recommended by the manufacturer (World Precision Instruments). In some experiments, maximal eNOS activity was determined in caveolin immunoprecipitates by measuring the conversion of $\left[{ }^{3} \mathrm{H}\right]$ arginine to $\left[{ }^{3} \mathrm{H}\right]$ citrulline, as described previously $(21)$. The results were calculated as $\mathrm{cpm} / \mu \mathrm{g}$ protein $/ \mathrm{min}$ after subtraction of the background signal obtained in the immunoprecipitate with an irrelevant $\operatorname{IgG}_{1}$. Data are presented for convenience as mean \pm SEM, and statistical analyses were made using Student's $t$ test or one-way ANOVA, where appropriate. $\left[{ }^{3} \mathrm{H}\right]$ cholesterol-labeled LDL preparation and uptake measurements. LDL was labeled to a final specific activity of $1-5 \times 10^{4} \mathrm{cpm} / \mu \mathrm{g}$ by equilibration with $1,2-\left[{ }^{3} \mathrm{H}\right]$ cholesterol-albumin-agarose complex, and the influx of LDL-derived $\left[{ }^{3} \mathrm{H}\right]$ cholesterol into BAEC monolayers was measured as reported by Fielding et al. (16). Briefly, cells were incubated at $37^{\circ} \mathrm{C}$ for 10 min with DMEM-LPDS containing $100-200 \mathrm{mg} / \mathrm{dl}\left[{ }^{3} \mathrm{H}\right]$ cholesterol-labeled LDL-C. Cells were then washed with ice-cold PBS, and adsorbed labeled LDL was successively displaced with an excess of unlabeled LDL-C with PBS containing $5 \mathrm{mg} / \mathrm{ml}$ albumin and with PBS alone; recovery of label was $>95 \%$, and influx was linear under these conditions. Cells were solubilized with liquid scintillation cocktail, and radioactivity was measured in a scintillation counter.

\section{Results}

Serum cholesterol levels and caveolin expression. Total cholesterol (TC) and LDL-C concentrations were determined in the plasma of normolipemic and untreated dyslipidemic volunteers. The values were distributed between three groups, identified as follows (Fig. 1a): normocholesterolemic (NC; $n=10, \mathrm{TC}<200 \mathrm{mg} / \mathrm{dl}$, and LDL-C $<130$ $\mathrm{mg} / \mathrm{dl}$ ); hypercholesterolemic (HC; $n=10$, TC > 240 $\mathrm{mg} / \mathrm{dl}$, and LDL-C > $160 \mathrm{mg} / \mathrm{dl}$ ); and an intermediate group (IC; $n=10)$. Nonconfluent BAEC were exposed to Dulbecco's modified Eagle's medium (DMEM) containing 50\% human serum and were grown for 48 hours. No significant modification in the abundance of eNOS protein was observed after incubation with the NC, IC, or HC serum (Fig. 1b, bottom), in agreement with the absence of changes in the cell number or total amounts of proteins between these three conditions (not shown). By contrast, caveolin abundance was dose-dependently augmented in endothelial cells exposed to increasing concentrations of 
a

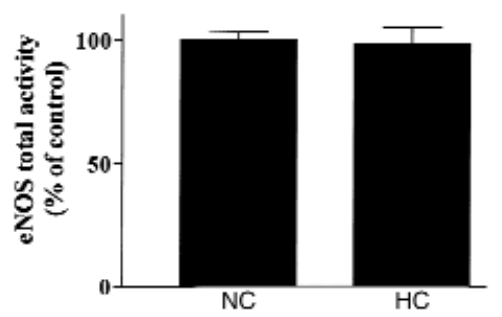

$\boldsymbol{b}$

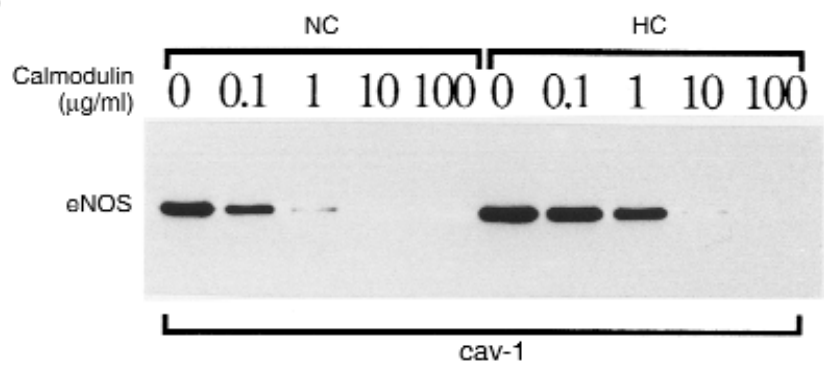

Figure 3

Release of eNOS from the caveolin immune complex by $\mathrm{Ca}^{2+}-\mathrm{CaM}$. Endothelial cells exposed for $48 \mathrm{~h}$ to $50 \%$ normocholesterolemic (NC) or hypercholesterolemic $(H C)$ human serum were collected, lysed, and solubilized as described in the text. (a) A bar graph (mean \pm SEM, $n=3$ ) illustrating the maximal eNOS enzyme activity, measured by the conversion of $\left[{ }^{3} \mathrm{H}\right]$ arginine in $\left[{ }^{3} \mathrm{H}\right]$ citrulline in the corresponding cell extracts immunoprecipitated with anti-caveolin-1 antibody. The data are expressed as percent of total eNOS activity in the immunoprecipitate obtained from cells exposed to NC serum. (b) Immunoblot with an anti-eNOS antibody of the same extracts immunoprecipitated with anti-caveolin-1 antibody and exposed, in presence of $\mathrm{Ca}^{2+}$, to increasing concentrations of exogenous CaM: 0, 0.1, 1, 10,100 $\mu \mathrm{g} / \mathrm{ml}$. The immune complexes bound to protein G-Sepharose beads were extensively washed in OG buffer, and the beads were then equally distributed in five separate aliquots. After $1 \mathrm{~h}$ incubation at $4^{\circ} \mathrm{C}$ in the presence of the indicated amounts of CaM, the beads were repelleted, the supernatant discarded, and the immune complex processed for SDS-PAGE and immunoblot analysis. These experiments were performed three times with similar results. CaM, calmodulin; OG, octylglucoside.

serum cholesterol by 1.7 -fold and 2.5 -fold in IC and HC groups $(P<0.01, n=6)$, respectively (Fig. $1 b$, top). Increases in caveolin abundance required at least 16-18 hours of exposure to high cholesterol levels and reached statistical significance after 30 hours of exposure.

In some experiments, cells were exposed to serum cholesterol in presence of the cysteine protease inhibitor $N$ acetyl-leu-leu-norleucinal (ALLN) in order to inhibit the catabolism of the soluble fragment of sterol regulatory element binding protein (SREBP) $(16,30)$. Figure $1 c$ shows that ALLN reduced caveolin expression in NC serum-treated endothelial cells and also inhibited the caveolin upregulation induced by HC serum exposure.

Serum cholesterol levels and the caveolin-eNOS heterocomplex. The increase in caveolin abundance prompted us to investigate the influence of high cholesterol exposure on the interaction between eNOS and caveolin. Figure $2 a$ (lanes 1 and 3) shows that in the absence of agonist exposure (basal state), caveolin antibodies immunoprecipitated both more caveolin (bottom) and more eNOS (top) from the lysates of endothelial cells exposed to high cholesterol levels. The absence of caveolin in the supernatant of these immunoprecipitations (IPs) (bottom, lanes 2 and 4) confirmed that the increase in immunoprecipitated caveolin from $\mathrm{HC}$ serum-treated cells quantitatively corresponded to the upregulation of the caveolin pool in these cells. By contrast, analysis of the eNOS fraction recovered in the IP supernatant (top, lanes 2 and 4) revealed significant differences between the two groups: despite the absence of any change in the total eNOS abundance between the different conditions (Fig. 1b), in extracts from NC serum-treated cells $\sim 20 \%$ of eNOS was recovered in the IP supernatant. However, there was no detectable signal for eNOS in the supernatant of the caveolin IP obtained from lysates of HC serum-treated cells. This suggested that at the basal level, the formation of the caveolin-eNOS complex, as measured by the fraction of immunoprecipitated eNOS, was quantitatively higher in endothelial cells exposed to high cholesterol concentrations. This observation was corroborated by the results obtained with endothelial cells treated with the calcium ionophore A23187, a receptor-independent agonist known to promote the binding of $\mathrm{Ca}^{2+}$-activated $\mathrm{CaM}$ to eNOS and the enzyme dissociation from caveolin (31). While in the NC serum-treated cells, all eNOS was now recovered in the supernatant of caveolin IP (Fig. 2a, top, lane 6), there was only a very small fraction $(-5 \%-10 \%)$ of eNOS detected in the IP supernatant recovered from lysates of $\mathrm{HC}$ serum-exposed cells (Fig. 2a, top, lane 8). The specificity of the caveolin IP in our experimental conditions was confirmed by the absence of immunoblotting signal for caveolin (and eNOS) when an irrelevant $\operatorname{IgG}_{1}$ was used for the IP (Fig. 2a, right). We have also found that in the endothelial cell type used in this study, most eNOS can be recovered in Percoll-isolated plasma membrane fractions (not shown). Using this detergent-free subfractionation technique in cells treated with A23187, the recovery of eNOS in the plasmalemmal pool was reduced to $10 \%$ in NC serum-treated cells, while in cells exposed to HC serum $>80 \%$ of eNOS remained at the plasma membrane. Finally, repeating the caveolin-eNOS coimmunoprecipitation experiments with the Percoll-isolated plasmalemmal fractions instead of total cell lysates consistently resulted in the same findings as presented in Fig. $2 a$ (not shown).

Serum cholesterol levels and the inbibitory caveolin-eNOS interaction. Because the interaction of caveolin with eNOS was shown to maintain the enzyme in its inactivated state (21-25), we next examined how this cholesterol-evoked increase in caveolin expression modulates $\mathrm{NO}$ production in endothelial cells. Quantification of NO release was obtained by direct measurement of $\mathrm{NO}$ concentration using an NO-specific amperometric probe positioned 0.1 $\mathrm{mm}$ above the cell monolayer. The calibration was performed on each experimental day, using SNAP as an NO donor; the calibration curve showed a linear relationship $\left(r^{2}=0.993\right)$ between NO concentration and the redox current $(\mathrm{pA})$, with an average sensitivity of $13.2 \pm 1.1 \mathrm{nM}$ $\mathrm{NO} / \mathrm{pA}$ (not shown). NO release in the incubation media was determined over a 5 -min period in the absence or presence of the calcium ionophore A23187 (5 $\mu \mathrm{M})$. In the non- 
stimulated conditions, the basal production of $\mathrm{NO}$ amounted to $23 \pm 4 \mathrm{pmol} / \mathrm{min} / \mathrm{mg}$ protein $(n=9)$. As shown in Fig. $2 b$, NC serum-treated endothelial cells showed a significantly higher basal level of NO production compared with cells incubated with the HC serum $(P<$ $0.05, n=9)$. Similarly, the effect of A23187 strikingly differed between the two conditions; in NC serum-treated cells, the NO production after A23187 stimulation amounted to $230 \pm 15 \%$ of the basal level. In cells exposed to $\mathrm{HC}$ serum, the A23187-evoked increase in NO production was attenuated to $60 \%$ over the basal level, a value corresponding to about half of the level reached in cells exposed to NC serum $(P<0.01, n=9)$.

Serum cholesterol levels and the allosteric regulation of caveolin-eNOS interaction by $\mathrm{Ca}^{+}-\mathrm{CaM}$. Although a highorder structure probably accounts for caveolin inhibition of eNOS activity, caveolin or peptides derived from its scaffolding domain are known to dramatically alter the enzyme's affinity constant for CaM $(21,24)$. We therefore examined whether the increase in caveolin abundance in HC serum-treated cells altered the titration of the caveolin-eNOS immune complex by $\mathrm{Ca}^{2+}-\mathrm{CaM}$ (Fig. 3). To attribute potential differences between the $\mathrm{NC}$ and $\mathrm{HC}$ treatments exclusively to changes in caveolin abundance, conditions were selected to ensure that caveolin immunoprecipitates contained similar absolute levels of eNOS; i.e., starting the IP protocol with $\sim 20 \%$ more lysates from NC serum-treated cells allowed the recovery of a similar amount of eNOS immunoprecipitated by caveolin antibodies. Figure $3 a$ shows that when maximal eNOS activity was determined by citrulline assay in the presence of a large excess of cofactors and CaM (to fully dissociate the heterocomplex), there was no significant difference in the enzyme activity of caveolin immunoprecipitates from extracts of $\mathrm{NC}$ or $\mathrm{HC}$ serum-treated cells. In these experimental conditions, however, striking differences were revealed in the titration of the caveolin-eNOS complex by CaM, as quantitated after caveolin IP from NC and $\mathrm{HC}$ serum-treated cell extracts. In the NC serum conditions (Fig. 3b), eNOS was completely displaced from caveolin with $10 \mu \mathrm{g} / \mathrm{ml} \mathrm{CaM}$, whereas a 10 -fold higher concentration $(100 \mu \mathrm{g} / \mathrm{ml})$ was required to dissociate the complex formed in HC serum-treated cells.

Lipoprotein subfractions and the caveolin-eNOS interaction. We next sought to determine which lipoprotein subfraction was responsible for the increase in both caveolin abundance and its inhibitory effect on eNOS enzyme activity. A density gradient ultracentrifugal procedure was used to isolate very-low-density lipoprotein (VLDL), LDL, $\mathrm{HDL}_{2}$, and $\mathrm{HDL}_{3}$ from human serum. Lipoproteindeficient human HC serum (LPDS) was also obtained from the same step gradient (see Methods) and was supplemented separately with each of the isolated lipoprotein subfractions in the same amount as originally present in the HC serum. As shown in Fig. 4a, the caveolin-eNOS association presented the same characteristics in endothelial cells exposed for 48 hours to $50 \%$ HC serum or $50 \%$ LPDS supplemented with $210 \mathrm{mg} / \mathrm{dl}$ LDL-C. Indeed, in both conditions eNOS appeared quantitatively associated with caveolin at the basal level (top, lanes 1 and 5) and was barely dissociated when cells were previously stimulated by the calcium ionophore A23187 (bottom, lanes 1, 2, 5, and 6 ). By contrast, when coimmunoprecipitation experiments were performed from endothelial cells exposed to VLDL, $\mathrm{HDL}_{2}$, and $\mathrm{HDL}_{3}$ (top and bottom, lanes 3, 4, 7-10), we observed that the caveolin-eNOS interaction was similar to that a

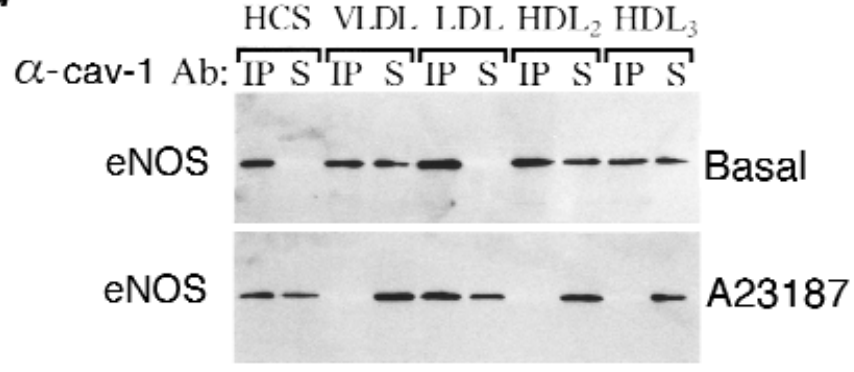

$\boldsymbol{b}$

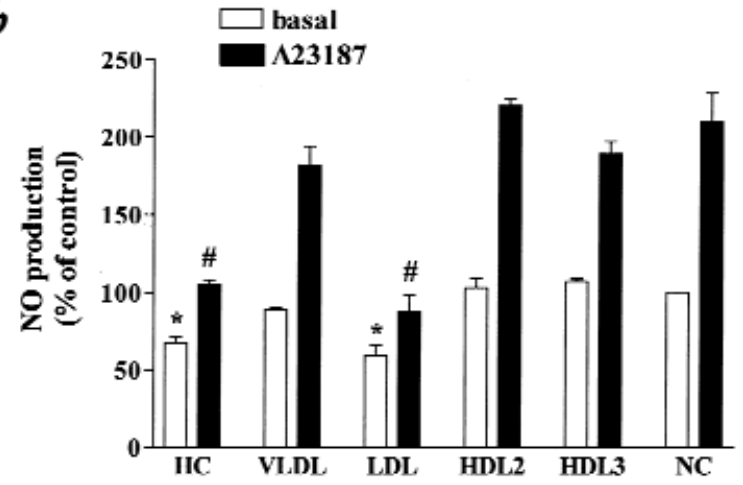

Figure 4

LDL, but not HDL or VLDL, fractions account for the caveolin-mediated attenuation of NO production in endothelial cells exposed to hypercholesterolemic human serum. Endothelial cells were exposed for $48 \mathrm{~h}$ to $50 \%$ hypercholesterolemic human serum or to $50 \%$ of LPDS supplemented with the indicated isolated lipoprotein fraction (cholesterol content: VLDL, $25 \mathrm{mg} / \mathrm{dl} ; \mathrm{LDL}, 210 \mathrm{mg} / \mathrm{dl} ; \mathrm{HDL}_{2}, 15 \mathrm{mg} / \mathrm{dl} ; \mathrm{HDL}_{3}, 25 \mathrm{mg} / \mathrm{dl}$ ). After a 5 -min incubation in the presence or absence of the calcium ionophore A23187 $(5 \mu \mathrm{M})$, cells were either tested for NO production or collected, lysed, and solubilized as described in the text. (a) Cells' extracts were immunoprecipitated with anti-caveolin-1 antibody, and both the immunoprecipitate (IP) and supernatant $(S)$ fractions were then separated on SDS-PAGE and immunoblotted with anti-eNOS antibody. Top: Immunoblot of IP and S fractions from nonstimulated cells. Bottom: Immunoblot of IP and S fractions from cells stimulated with A23187. Note that to obtain the S fractions, the supernatants from the IP were immunoprecipitated with anti-eNOS antibody in order to detect quantitatively the amount of eNOS left behind the initial caveolin IP. (b) Shown is a bar graph (mean \pm SEM, $n=3$ ) illustrating the NO production (measured with a microsensor) at the basal level (open bars) and after exposure to A23187 (filled bars) in cultures of endothelial cells exposed to hypercholesterolemic (HC) human serum or to specific lipoprotein fractions as indicated; the data are expressed as percent of NO production in nonstimulated cells exposed to NC serum. ${ }^{*} P<0.05$ vs. basal conditions with $\mathrm{NC}$, VLDL, $\mathrm{HDL}_{2}$, and $\mathrm{HDL}_{3} .{ }^{\#} P<0.01$ vs. stimulated conditions with $\mathrm{NC}, \mathrm{VLDL}, \mathrm{HDL}_{2}$, and $\mathrm{HDL}_{3}$. HDL, high-density lipoprotein; LPDS, lipoprotein-deficient human serum; $V L D L$, very-low-density lipoprotein. 
observed in the NC serum-exposed cells (Fig. 2): eNOS was only partially associated to caveolin at the basal level and was completely dissociated from the caveolin complex after A23187-evoked $\mathrm{Ca}^{2+}$ increase.

We next measured the NO production in cells exposed to LPDS supplemented with each lipoprotein subfraction (Fig. 4b). Measurements of NO release revealed that the LDL subfraction alone mimicked the effect obtained with the HC serum: basal and stimulated NO production were inhibited when compared with the results obtained from endothelial cells exposed to the NC serum or to LPDS supplemented with any of the non-LDL subfractions (Fig. $4 b$ ). The ratio of stimulated vs. basal NO production, which was 2.1:1 in cells exposed to NC serum, was attenuated to 1.6:1 and 1.5:1 in cells exposed to HC and to the corresponding LDL subfraction, respectively.

Dose dependence for the stabilization of the caveolin-eNOS inhibitory complex by $L D L$. By exposing endothelial cells to LPDS containing increasing concentrations of LDL-C (100-200 mg/dl), we next examined the dose dependence in both the stabilization of the caveolin-eNOS complex as well as the inhibitory effect on NO release. We found that while eNOS expression was not altered by the different LDL treatments (Fig. 5a, bottom), caveolin abundance was dosedependently increased by LDL-C (Fig. $5 a$, middle). In parallel to the increase in caveolin expression, we found that in nonstimulated cells the association between both proteins, as reflected by the fraction of eNOS immunoprecipitated by caveolin antibodies, augmented proportionally with the LDL content in the culture medium (Fig. $5 a$, top left). As a corollary, less eNOS was dissociated from caveolin (i.e., a higher fraction of eNOS was recovered in the caveolin IP) after A23187 stimulation in endothelial cells exposed to higher $(>150 \mathrm{mg} / \mathrm{dl}$ ) LDL concentrations (Fig. $5 a$, top right).

We next examined the correlation between the caveolin-eNOS heterocomplex formation and NO production after treatment of endothelial cells with LDL. NO production from cells treated with $100 \mathrm{mg} / \mathrm{dl}$ LDL-C (i.e., the minimal LDL concentration in our experimental conditions) was augmented by 2.2-fold after A23187 stimulation when compared with the basal release of NO (Fig. $5 b$ ). Increasing LDL-C concentrations led to a dosedependent decrease in basal and A23187-stimulated NO production (Fig. 5b); after incubation with $200 \mathrm{mg} / \mathrm{dl}$ LDL-C (i.e., the maximal LDL concentration in our experimental conditions), the ratio of stimulated vs. nonstimulated NO release was reduced to $1.5(P<0.01)$.

Using the same protocol of cell exposure to increasing doses of LDL-C, we next examined the correlation between caveolin abundance and cholesterol uptake in BAEC. Cholesterol uptake was measured by exposing the cells for 10 minutes to DMEM-LPDS containing 100-200 $\mathrm{mg} / \mathrm{dl}\left[{ }^{3} \mathrm{H}\right]$ cholesterol-labeled LDL-C . Figure $5 c$ shows a linear correlation $\left(r^{2}=0.964\right)$ between the increase in cholesterol uptake and the expression of caveolin.

To more directly assess the importance of LDL's oxidizing ability as a determinant of the responses observed in our model, we tested the effects of antioxidant treatments on the upregulation of caveolin and caveolin-eNOS interaction in cells treated with LDL-C. As illustrated in Fig. $5 d$ (top), cotreatment of endothelial cells with the antioxidant DTPA for the entire LDL incu- bation time had no effect on the upregulation of caveolin abundance induced by high LDL-C. Also, DTPA treatment had no effect on the pattern of eNOS association to caveolin, as assayed by coimmunoprecipitation in extracts of endothelial cells stimulated with A23187 at 100 or $200 \mathrm{mg} / \mathrm{dl}$ LDL-C (Fig. $5 d$, bottom; compare with Fig. 5a, top, in the absence of DTPA). High LDL-C treatment, which did not alter the eNOS abundance, still increased the fraction of eNOS immunoprecipitated by anti-caveolin antibodies (reflecting increased caveolin-eNOS heterocomplex formation), despite the $\mathrm{Ca}^{2+}$ ionophore treatment. Similar results were obtained by using $20 \mu \mathrm{M}$ BHT or $100 \mu \mathrm{g} / \mathrm{ml}$ copper/zinc superoxide dismutase $(\mathrm{Cu} / \mathrm{Zn} \mathrm{SOD})$ instead of DTPA.

\section{Discussion}

Hypercholesterolemia is associated with an impaired endothelial NO production, and as a consequence, alterations in eNOS abundance and activity were proposed to constitute early events in the development of atherosclerosis (5-14). In this study, we provide biochemical and functional evidence that high levels of LDL-C decrease the production of NO in endothelial cells by upregulating the abundance of the structural protein caveolin and promoting its inhibitory interaction with eNOS. Our results identify caveolin as a cholesterol-sensor protein linking the plasma concentration of LDL-C to the basal- and agonist-stimulated production of NO.

Based on several independent experimental approaches $(21-25,31,32)$, a model for the eNOS activation-deactivation process was recently proposed for the agonist stimulation of $\mathrm{NO}$ production in endothelial cells. According to this model, when intracellular $\mathrm{Ca}^{2+}$ concentration is increased, a caveolin-eNOS regulatory cycle (25, 31 ) is initiated wherein (a) $\mathrm{Ca}^{2+}-\mathrm{CaM}$ activates eNOS by disrupting the heteromeric complex formed between eNOS and caveolin; (b) activated eNOS translocates from caveolae; and (c) $\mathrm{Ca}^{2+}$ returns to basal levels, and CaM dissociates from eNOS, which may then reform a complex with caveolin and is retargeted to plasmalemmal caveolae. Thus, this dynamic equilibrium could be modulated by any stimulus mobilizing $\mathrm{Ca}^{2+}$ in the vicinity of caveolae, as well as by changes in the abundance of either of the two enzyme's partners, namely caveolin and CaM. In the current report, caveolin-eNOS coimmunoprecipitation experiments provided evidence that the upregulation of caveolin in native endothelial cells promoted the displacement of this dynamic equilibrium toward the stabilization of the inhibitory caveolin-eNOS complex. Furthermore, CaM titration of the caveolin-eNOS immune complex showed that more $\mathrm{Ca}^{2+}-\mathrm{CaM}$ was required to compete with the higher amounts of caveolin interacting with eNOS in endothelial cells exposed to HC serum. The CaM dose-dependent release of eNOS from the caveolin immunoprecipitate is in agreement with the model of mutually exclusive interaction of caveolin and $\mathrm{Ca}^{2+}-\mathrm{CaM}$ with eNOS. Thus, although other components of the caveolin heterocomplex, such as hsp90 (33), have been identified as modulators of eNOS activity and targeting, the competition between CaM and caveolin appears to be a major determinant of the alteration of eNOS properties by high cholesterol after 
calcium mobilization, at least in our cell model. Importantly, the use of the NO-selective microprobe allowed us to demonstrate that upon small changes in the extracellular LDL concentrations, limited but significant variations in the cellular caveolin content could have profound effects on basal and stimulated $\mathrm{NO}$ release through stabilization of the caveolin-eNOS inhibitory complex. In the endothelial cells used in this study, most of eNOS is located at the plasma membrane (see Results and ref. 34), implicating this caveolar pool of eNOS as a main target for inhibitory interaction with caveolin. In other experimental models, however, caveolin and eNOS are found both in plasmalemmal and nonplasmalemmal cell compartments (35), suggesting that the proportion of the caveolin-eNOS heterocomplex within caveolae may vary according to the cell origin and/or phenotype. These limitations aside, this study is, to our knowledge, the first demonstration that the inhibitory interaction with an enzyme (eNOS) occurs upon such changes in caveolin abundance as those induced by pathophysiologically relevant interventions in intact cells, i.e., exposure to high cholesterol levels.

Several lines of evidence indicate that the upregulation of caveolin abundance in endothelial cells exposed to high levels of native LDL-C directly results from the increase in caveolin gene transcription induced by the increased cell content in cholesterol. Bist et al. (17) had identified two sterol regulatory element (SRE)-like sequences in the caveolin promoter that mediate the inhibition of caveolin transcription upon binding of SREBP-1 fragments. Because FC inhibits the cleavage of SREBP (required for its activation), these authors showed that exposure of fibroblasts to an $\sim 10$-fold increase in extracellular cholesterol content results in an $\sim 4$-fold increase in caveolin mRNA levels $(16,17)$. Similarly, we observed a strong correlation between choles- $a$

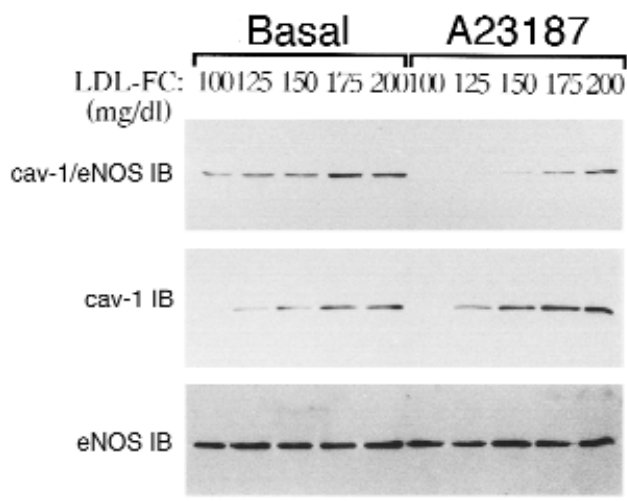

$b$

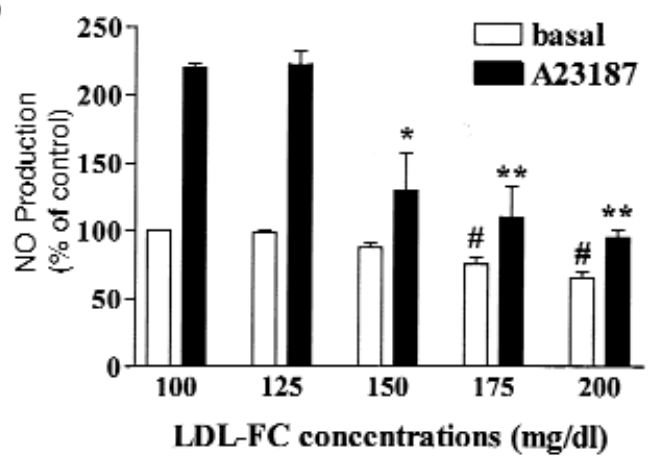

c

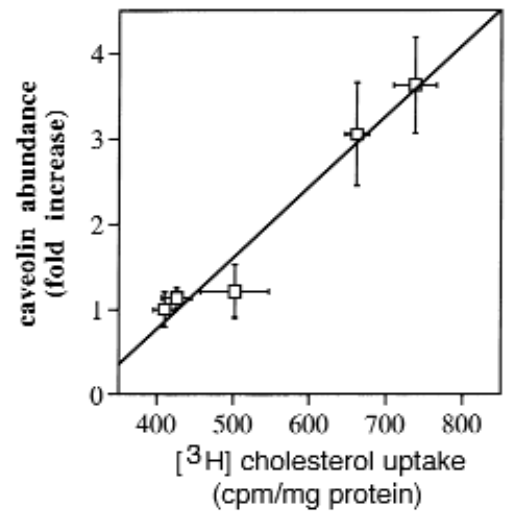

$d$

Figure 5

Dose-dependent increase in caveolin expression and stabilization of the caveolin-eNOS interaction by LDL fractions. Endothelial cells were exposed for $48 \mathrm{~h}$ to $50 \%$ LPDS with the indicated concentrations of LDL-C. After 5-min incubation in the presence or absence of the calcium ionophore A23187 (5 $\mu \mathrm{M})$, cells were either tested for NO production or collected, lysed, and solubilized as described in the text. (a) Top: Extracts from A23187treated and untreated cells were immunoprecipitated with anti-caveolin-1 antibody, and the IP fraction was separated on SDS-PAGE and immunoblotted with an anti-eNOS antibody. The extracts from the corresponding cells were also separated on SDS-PAGE and immunoblotted with either an anti-caveolin (middle) or anti-eNOS antibody (bottom). Note that longer exposure of the same blot also reveals detectable caveolin at $100 \mathrm{mg} / \mathrm{dl}$ LDL-C. These experiments were performed two times with similar results. (b) A bar graph (mean $\pm \mathrm{SEM}, n=3$ ) illustrating the NO production (measured with a microsensor) at the basal level (open bars) and after exposure to A23187 (filled bars) in cultures of endothelial cells previously incubated in presence of the indicated amounts of LDL; the data are expressed as percent of NO production in nonstimulated cells exposed to $100 \mathrm{mg} / \mathrm{dl}$ LDL-C. ${ }^{\#} P<0.01 \mathrm{vs}$. basal $100 \mathrm{mg} / \mathrm{dl} \mathrm{LDL}$ and $125 \mathrm{mg} / \mathrm{dl} \mathrm{LDL}$ conditions. ${ }^{*} P<0.05$, ${ }^{*} P<0.01 \mathrm{vs}$. stimulated $100 \mathrm{mg} / \mathrm{dl}$ $\mathrm{LDL}$ and $125 \mathrm{mg} / \mathrm{dl} \mathrm{LDL}$ conditions. (c) Relationship between cholesterol uptake and caveolin abundance. Individual values were obtained from endothelial cells exposed to 100, 125, 150, 175, and $200 \mathrm{mg} / \mathrm{dl}$ LDL-C. (d) Top: Extracts from cells incubated with $100 \mathrm{or} 200 \mathrm{mg} / \mathrm{dl}$ LDL-C in presence of an antioxidant, DTPA $(50 \mu \mathrm{M})$, and acutely treated with $5 \mu \mathrm{M} \mathrm{A23187} \mathrm{were} \mathrm{separated} \mathrm{on} \mathrm{SDS-PAGE} \mathrm{and} \mathrm{immunoblotted} \mathrm{with} \mathrm{anti-caveolin}$ antibody (two lanes per condition). Bottom: The corresponding extracts were also immunoprecipitated with anti-eNOS (lanes 1 and 3 ) or anti-caveolin antibody (lanes 2 and 4), and the IP fraction was separated on SDS-PAGE and immunoblotted with an anti-eNOS antibody. These experiments were performed two times with similar results. DTPA, diethylenetriamine pentaacetic acid. 
terol uptake and caveolin abundance over the range of LDL-C (100-200 mg/dl) used in our experiments (Fig. $5 c$ ). Moreover, using the cysteine protease inhibitor ALLN to reduce the catabolism of SREBP, we demonstrated its superinhibitory role on caveolin gene transcription in our endothelial cell model. Altogether, our experiments confirm the cholesterol-dependent upregulation of caveolin at the protein level and provide evidence that pathological alterations of the caveolin pool in endothelial cells may have dramatic consequences on the regulation of eNOS and potentially other signaling molecules known to bind caveolin.

Numerous studies exploring the proatherogenic risk factors in human and animal vessels have focused on the decrease in bioavailability of NO because of its augmented degradation by superoxide, a species known to be elevated in various cardiovascular disease states (reviewed in refs. 9, 10). Hypercholesterolemia is known to be associated with an elevation in superoxide production from various sources, including xanthine oxidase, NADPH-oxidase, and eNOS itself $(6,9)$. In this study, NO detection was performed in the presence of an excess of superoxide dismutase, thereby excluding the oxidative stress as a potential cause of the impairment in NO production by $\mathrm{HC}$ serum-treated cells in our experimental conditions. Aside from the inactivation of NO by reactive oxygen species, several studies examined the relationship between dyslipidemia and endothelial dysfunction through a direct modulation of eNOS expression and/or activation; they identified defects in the coupling of the receptor-G protein interaction leading to eNOS stimulation $(6,36)$ and effects of oxidized LDL (oxLDL) on the steady-state mRNA levels of the enzyme $(13,14)$. In the present study, these mechanisms are unlikely to account for the impairment in eNOS activity observed in endothelial cells exposed to HC serum or to high concentrations of isolated LDL. First, we examined the stimulation of eNOS by a receptor-independent agonist (i.e., the calcium ionophore A23187) to exclude the $G$ protein-coupled receptor signaling cascade (leading to eNOS activation) as a potential target of the high cholesterol treatment. Second, the use of LDL subfractions isolated from either $\mathrm{HC}$ or NC human serum led to the same dose-dependent inhibition of $\mathrm{NO}$ release, indicating that at least the oxidizability of LDL does not account for the differences observed, as corroborated by direct measurements of minimally modified LDL in our fractions. Third, Fielding et al. (16) demonstrated that caveolin mRNA levels are upregulated by FC but downregulated by oxysterols in cell cultures. Accordingly, exposure of our endothelial cell cultures to high concentrations of LDL-C consistently produced an upregulation of caveolin expression that we have demonstrated to be abrogated by an inhibitor of SREBP catabolism (see above), suggesting that products derived from LDL oxidation have negligible effects on caveolin protein in our experimental conditions. Moreover, the use of extracellular antioxidants such as DTPA, BHT, or $\mathrm{Cu} / \mathrm{Zn}$ SOD, which were maintained during the whole cell exposure to high cholesterol levels, had no effect on the increase in caveolin abundance or stabilization of the caveolin-eNOS interaction as observed in the absence of antioxidants.

In conclusion, this study provides a new pathogenic mechanism linking high LDL-C levels to the decrease in basal and stimulated NO production through alteration in eNOS activity. Importantly, this impairment in NO release by endothelial cells occurs in the absence of any change in eNOS abundance and may, therefore, be one of the early events among the proatherogenic processes induced by hypercholesterolemia. Accordingly, the primitive deficit in peripheral artery vasodilation associated with high plasma cholesterol is generally observed in the absence of changes in eNOS protein levels $(6,37)$ or structural alterations of the vascular wall $(3,4)$. Therefore, regulation of eNOS activity at the posttranslational level appears to play a prominent role in these early steps of atherogenesis. More generally, this study identifies a key role for variations of caveolin abundance in modulating the catalytic activity of signaling enzymes and provides new perspectives for therapeutic interventions that may arise from a better understanding of the regulation of caveolin expression in various cardiovascular diseases.

\section{Acknowledgments}

The authors thank Benoit Boland from the General Internal Medicine Unit at the Clinique Universitaire St. Luc (Université catholique de Louvain) for his help in the collection of hypercholesterolemic sera in the Lipid Clinic. We are grateful to Paul Holvoet (Center for Molecular and Vascular Biology, Katholieke Universiteit Leuven, Belgium) for his help in measuring levels of modified LDL in human serum by ELISA. This work was supported by a grant (Action de Recherche Concertée 96/01-199) from the French Community of Belgium (to J.-L. Balligand), and by grants from the Belgian National Fund for Scientific Research (FRFC grant 2.4611.99 to O. Feron; and FRSM 3.4537.98 and 9.4550.97 to J.-L. Balligand). O. Feron is a Senior Research Assistant from the Belgian National Fund for Scientific Research.

1. Ross, R. 1993. The pathogenesis of atherosclerosis: a perspective for the 1990s. Nature. 362:801-809.

2. Lee, R.T., and Libby, P. 1997. The unstable atheroma. Arterioscler. Thromb. Vasc. Biol. 17:1859-1867.

3. Seiler, C., Hess, O.M., Buechi, M., Suter, T.M., and Krayenbuehl, H.P. 1993. Influence of serum cholesterol and other coronary risk factors on vasomotion of angiographically normal coronary arteries. Circulation. 88:2139-2148.

4. Reddy, K.G., Nair, R.N., Sheehan, H.M., and Hodgson, J.M. 1994. Evidence that selective endothelial dysfunction may occur in the absence of angiographic or ultrasound atherosclerosis in patients with risk factors for atherosclerosis. J. Am. Coll. Cardiol. 23:833-843.

5. Creager, M.A., et al. 1990. Impaired vasodilation of forearm resistance vessels in hypercholesterolemic humans. J. Clin. Invest. 86:228-234.

6. Flavahan, N.A. 1992. Atherosclerosis or lipoprotein-induced endothelial dysfunction: potential mechanisms underlying reduction in EDRF/NO activity. Circulation. 85:1927-1938.

7. Casino, P.R., Kilcoyne, C.M., Quyyumi, A.A., Hoeg, J.M., and Panza, J.A. 1993. The role of nitric oxide in endothelium-dependent vasodilation in hypercholesterolemic patients. Circulation. 88:2541-2547.

8. Balligand, J.L., and Cannon, P.J. 1997. Nitric oxide synthases and cardiac muscle: autocrine and paracrine influences. Arterioscler. Thromb. Vasc. Biol. 17:1846-1858.

9. Harrison, D.G. 1997. Cellular and molecular mechanisms of endothelial cell dysfunction. J. Clin. Invest. 100:2153-2157.

10. Wever, R., Stoes, E., and Rabelink, T.J. 1998. Nitric oxide and hypercholesterolemia: a matter of oxidation and reduction? Atherosclerosis. 137(Suppl.):51s-60s.

11. Jacobs, M., Plane, F., and Bruckdorfer, K.R. 1990. Native and oxidized lowdensity lipoproteins have different inhibitory effects on endotheliumderived relaxing factor in the rabbit aorta. Br. J. Pharmacol. 100:21-26.

12. Galle, J., Mulsch, A., Busse, R., and Bassenge, E. 1991. Effects of native and oxidized low density lipoproteins on formation and inactivation of endothelium-derived relaxing factor. Arterioscler. Thromb. 11:198-203.

13. Liao, J.K., Shin, W.S., Lee, W.Y., and Clark, S.L. 1995. Oxidized low-den- 
sity lipoprotein decreases the expression of endothelial nitric oxide synthase. J. Biol. Chem. 270:319-324.

14. Hirata, K., et al. 1995. Low concentrations of oxidized low-density lipoprotein and lysophosphatidylcholine upregulate constitutive nitric oxide synthase mRNA in bovine aortic endothelial cells. Circ. Res. 76:958-962.

15. Fielding, C.J., and Fielding, P.E. 1997. Intracellular cholesterol transport. J. Lipid. Res. 38:1503-1521.

16. Fielding, C.J., Bist, A., and Fielding, P.E. 1997. Caveolin mRNA are upregulated by free cholesterol and down-regulated by oxysterols in fibroblast monolayers. Proc. Natl. Acad. Sci. USA. 94:3753-3758.

17. Bist, A., Fielding, P.E., and Fielding, C.J. 1997. Two sterol regulatory element-like sequences mediate up-regulation of caveolin gene transcription in response to low density lipoprotein free cholesterol. Proc. Natl. Acad. Sci. USA. 94:10693-10698.

18. Shaul, P.W., et al. 1996. Acylation targets endothelial nitric-oxide synthase to plasmalemmal caveolae. J. Biol. Chem. 271:6518-6522.

19. Feron, O., et al. 1996. Endothelial nitric oxide synthase targeting to caveolae: specific interactions with caveolin isoforms in cardiac myocytes and endothelial cells. J. Biol. Chem. 271:22810-22814.

20. Okamoto, T., Schlegel, A., Scherer, P.E., and Lisanti, M.P. 1998. Caveolins, a family of scaffolding proteins for organizing "preassembled signaling complexes" at the plasma membrane. J. Biol. Chem. 273:5419-5422.

21. Michel, J.B., Feron, O., Sacks, D., and Michel, T. 1997. Reciprocal regulation of endothelial nitric-oxide synthase by $\mathrm{Ca}^{2+}$-calmodulin and caveolin. J. Biol. Chem. 272:15583-15586.

22. Ju, H., Zou, R., Venema, V.J., and Venema, R.C. 1997. Direct interaction of endothelial nitric-oxide synthase and caveolin-1 inhibits synthase activity. J. Biol. Chem. 272:18522-18525.

23. Garcia-Cardena, G., et al. 1997. Dissecting the interaction between nitric oxide synthase and caveolin. J. Biol. Chem. 272:25437-25440.

24. Michel, J.B., Feron, O., Sase, K., Prabhakar, P., and Michel, T. 1997. Caveolin versus calmodulin: counterbalancing allosteric modulators of nitric oxide synthase. J. Biol. Chem. 272:25907-25912.

25. Michel, T., and Feron, O. 1997. Nitric oxide synthases: which, where, how and why? J. Clin. Invest. 100:2146-2152.
26. Chapman, M.J., Goldstein, S., Lagrange, D., and Laplaud, P.M. 1981. A density gradient ultracentrifugal procedure for the isolation of major lipoprotein classes from human serum. J. Lipid Res. 22:339-358.

27. Holvoet, P., Vanhaecke, J., Janssens, S., Van de Werf, F., and Colen, D. 1998. Oxidized LDL and malondialdehyde-modified LDL in patients with acute coronary syndromes and stable coronary artery disease. Circulation. 98:1487-1494.

28. Balligand, J.L., et al. 1994. Cytokine-inducible nitric oxide synthase (iNOS) expression in cardiac myocytes. Characterization and regulation of iNOS expression and detection of iNOS activity in single cardiac myocytes in vitro. J. Biol. Chem. 269:27580-27588.

29. Gauthier, C. et al. 1998. The negative inotropic effect of beta3-adrenoceptor stimulation is mediated by activation of a nitric oxide synthase pathway in human ventricle. J. Clin. Invest. 102:1377-1384.

30. Wang, X., Sato, R., Brown, M.S., Hua, X., and Goldstein, J.L. 1994. SREBP-1, a membrane-bound transcription factor released by sterol-regulated proteolysis. Cell. 77:53-62.

31. Feron, O., Saldana, F., Michel, J.B., and Michel, T. 1998. The eNOS-caveolin regulatory cycle. J. Biol. Chem. 273:3125-3128.

32. Feron, O., Michel, J.B., Sase, K., and Michel, T. 1998. Dynamic regulation of endothelial nitric oxide synthase: complementary roles of dual acylation and caveolin interactions. Biochemistry. 37:193-200.

33. Garcia-Cardena, G., et al. 1998. Dynamic activation of endothelial nitric oxide synthase by Hsp90. Nature. 392:821-824.

34. Prabhakar, P., et al. 1998. Receptor-regulated translocation of endothelial nitric-oxide synthase. J. Biol. Chem. 273:27383-27388.

35. Garcia-Cardena, G., Oh, P., Liu, J., Schnitzer, J.E., and Sessa, W.C. 1996. Targeting of nitric oxide synthase to endothelial cell caveolae via palmitoylation: implications for nitric oxide signaling. Proc. Natl. Acad. Sci. USA. 93:6448-6453.

36. Liao, J.K., and Clark, S.L. 1995. Regulation of G protein alpha (i2) subunit expression by oxidized low-density lipoprotein. J. Clin. Invest. 95:1457-1463.

37. Kanazawa, K., et al. 1996. Endothelial constitutive nitric oxide synthase protein and mRNA increased in rabbit atherosclerotic aorta despite impaired endothelium-dependent vascular relaxation. Am. J. Pathol. 148:1949-1956. 